Pathologe 2020 - 41:561-562

https://doi.org/10.1007/s00292-020-00846-0

(c) Springer Medizin Verlag $\mathrm{GmbH}$, ein Teil von Springer Nature 2020

\section{FISH, CISH, SISH, DDISH, RNA-in- situ-Hybridisierung ... braucht man das (noch)?}

Die molekularpathologische Diagnostik ist im Umbruch und im Aufbruch. Viele faszinierende neue Methoden und Verfahren haben unser diagnostisches Spektrum erheblich erweitert. Große Multiplex-Sequenzierpanels auf DNA- und RNA-Ebene erlauben eine präzise Diagnostik von Mutationen, Genfusionen, Spleißvarianten in hoher Auflösung, faszinierender Präzision und bis in den Bereich von sehr geringen Allelfrequenzen. Viele Untersuchungen lassen sich auch an Liquid Biopsies, nicht nur aus Blut, sondern auch aus Ergussflüssigkeiten sehr präzise durchführen. Hochsensitive Verfahren wie die Digital-dropletPCR detektieren Varianten auch weit unter der $1 \%$-Allelfrequenz-Grenze. Neben den genomischen Untersuchungen sind auch die epigenetischen Analysen, insbesondere Methylierungsassays bereits diagnostischer Standard.

Multiplex-Genexpressionsassays, die mehrere hundert Gene simultan an formalinfixiertem, paraffineingebettetem Material (FFPE-Material) untersuchen können, stehen für Forschungsfragestellung zur Verfügung. Multiplex-Immunhistochemie gibt Antworten auf Fragen zur räumlichen Interaktion von Zellpopulationen im Gewebe. Digitale Bildauswertung ist für quantitative Analysen verfügbar und die Möglichkeiten künstlicher Intelligenz bei der Analyse von HE-Präparaten scheinen schier unerschöpflich zu sein.

Neben diesen Hochdurchsatz- und Multiplexverfahren behauptet sich die

Hans-Ulrich Schildhaus

Institut für Pathologie, Universitätsklinikum Essen, Essen, Deutschland

\title{
In-situ-Hybridisierung in der molekularpathologischen Diagnostik
}

In-situ-Hybridisierung (ISH) in ihren diagnostischen Anwendungen. Auch viele Institute, die über die neuesten Sequenzierverfahren verfügen, führen aktuell mehr Fluoreszenz-in-situ-Hybridisierungs(FISH)-Analysen durch denn je.

Die Methode hat ja auch ihre Vorteile:

- ISH ist einfach. Einfach in der Durchführung im Labor und einfach in der Auswertung.

- ISH ist diagnostisch relevant. Eine Reihe von klinisch bedeutsamen Diagnosen, insbesondere auf dem Gebiet der Lymphom- und Sarkomdiagnostik sind präzise mit ISH zu stellen (siehe auch die beiden Beiträge zu diesen Themengebieten in diesem Heft).

- ISH ist schnell. Nur wenige molekularpathologische Verfahren erlauben eine valide Diagnostik innerhalb von 1-2 Arbeitstagen, wie sie mittels ISH regelhaft möglich ist. Auch die Auswertung eines qualitativ guten FISHPräparates ist schnell getan: Wenige Minuten Analysezeit reichen in der Regel aus.

- ISH ist morphologisch. Analysen werden jederzeit unter morphologischer Kontrolle durchgeführt. Intratumorale Heterogenitäten können mit dieser Methode optimal adressiert werden.

Methodenspezifische Expertise ist natürlich auch hier erforderlich - wie bei jeder molekularpathologischen Analyse. Dazu gehört auch das Wissen um die Grenzen des Verfahrens, aber natürlich auch die Freude am diagnostischen Gelingen, die sich besonders schnell in der
FISH oder ihren verwandten methodischen Abwandlungen einstellen kann. Für einige diagnostische Fragestellungen, zum Beispiel die HER2-Diagnostik (auch hierzu findet sich ein umfangreicher Übersichtsartikel im Heft) oder die großzelligen Lymphome, ist (F)ISH nach wie vor die Referenzmethode der Wahl.

Seit den ersten klinisch-diagnostischen Anwendungen, die im Zusammenhang mit dem Nachweis des Philadelphia-Chromosoms und der bcr-ablTranslokation aufkamen, hat sich die Methode erheblich entwickelt und verbessert. Kommerziell verfügbare Sonden sind lichtstärker, die Hybridisierungssignale sind bei den gängigen Assays viel besser vom Hintergrund abzugrenzen und somit einfacher auszuwerten. Verbesserte Reagenzien und Protokolle sowie die Möglichkeit zur Automatisierung der Methode tragen ferner zur Sicherheit und Präzision der Technik bei. Auswerteassistenzsysteme können digitalisierte FISH-Präparate bereits analysieren oder bei der Auswertung helfen. Mehrfarbige Assays mit 3 oder 4 Fluorchromen erlauben die simultane Analyse von mehr als einem Gen und somit durchaus die Beantwortung komplexer Fragestellungen. Ein sich besonders schnell entwickelnder, noch vergleichsweise neuer Zweig ist ferner die RNA-in-situ-Hybridisierung (der Methodik und ihren Anwendungsgebieten ist ein eigener Beitrag in diesem Heft gewidmet). Dieses Verfahren hat aktuell seinen Stellenwert im Bereich von klinischer Forschung. Vor allem immer dann, wenn diagnostisch valide Antikörper für Immunhistochemie noch nicht verfügbar sind. Allerdings ist auch diese Methode multiplexfähig 


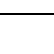

Aktuelle Informationen

und erlaubt dann sehr komplexe Analysen unter Berücksichtigung räumlicher Aspekte im Gewebe. Diese Methode hat sicher ein erhebliches Potenzial auch in klinische Anwendungen zu kommen. Verwandte Verfahren sind seit längerem beispielsweise zum Erregernachweis im diagnostischen Gebrauch.

Die In-situ-Hybridisierung ist also ein Verfahren mit Tradition, aber auch mit aktuellen Neuentwicklungen. Sie behauptet ihre methodischen Einsatzgebiete und gewinnt neue dazu, die vor allem durch die Vielzahl von neuen Genfusionen gespeist werden, die nahe$\mathrm{zu}$ wöchentlich als Ergebnis großer Sequenzierstudien publiziert werden. Auch im Bereich der Detektion von Genkopiezahlveränderungen („copy number variations", CNV) bei Tumoren spielt ISH weiter eine relevante Rolle. Medikamentenzulassungen, die auf $\mathrm{CNV}$ beruhen, verlangen bislang stets den ISH-Nachweis.

In diesem Themenheft, das sich ungewöhnlicherweise einer bestimmten Technik widmet, haben wir versucht, einmal den Bogen von methodischen Aspekten bis zu allgemein relevanten klinischen Anwendungen zu spannen. Ein Grundlagen-orientierter Beitrag greift die besonderen Aspekte der RNA-basierten Anwendungen auf. In weiteren Artikeln werden klinische Anwendungsgebiete exemplarisch dargestellt. Darunter natürlich HER2 - gewissermaßen der Prototyp der (F)ISH-basierten prognostischen und prädiktiven Diagnostik überhaupt. In weiteren Artikeln werden organ- bzw. tumortypbezogene Anwendungen genauer beleuchtet: Die FISHDiagnostik für prädiktive Biomarker bei Lungenkarzinomen, die diagnostischen Biomarker in der Sarkompathologie sowie die Anwendungen der FISH zur Klassifikation von Lymphomen, die vorrangig prognostische Relevanz hat. Die letztgenannten Artikel zeigen eindrücklich, wie sehr molekularpathologische Diagnostik davon abhängig ist, dass Untersuchungsergebnisse in einen klinischen und morphologischen Kontext eingebettet werden. Auch dies ist ein Erfordernis, das die In-situ-Hybridisierung mit praktisch allen anderen molekularpathologischen Anwendungen gemeinsam hat. Die sehr verantwortungsvolle Diagnostik, insbesondere die ISH im Rahmen prädiktiver Biomarkeranalysen, verlangt naturgemäß eine rigorose Qualitätskontrolle. In diesem Zusammenhang hat sich die Qualitätssicherungs-Initiative Pathologie (QuIP) dankenswerterweise seit vielen Jahren auch um die In-situHybridisierung bemüht. Aus der retrospektiven Analyse der Ringversuche zu diesem Thema lässt sich viel lernen; daher widmet sich ein Artikel dezidiert diesem Thema.

Dieses Heft ist entstanden durch die fleißige und kenntnisreiche Arbeit, die Kolleginnen und Kollegen in die Erstellung der Beiträge gesteckt haben. Ich möchte daher allen herzlich danken, die mit ihren Beiträgen diese Übersicht ermöglicht haben. Ferner gebührt mein Dank auch den Reviewerinnen und Reviewern, die die Artikel durchgesehen und zu Verbesserungen beigetragen haben.

Mit diesen Vorbemerkungen verbinde ich die Hoffnung, dass Ihnen, liebe Leserin, lieber Leser, dieses Themenheft Erkenntnisgewinn bescheren möge.

\section{Herzlich}

Ihr

Hans-Ulrich Schildhaus

\section{Korrespondenzadresse}

Univ.-Prof. Dr. med. Hans-Ulrich Schildhaus Institut für Pathologie, Universitätsklinikum Essen

Hufelandstraße 55, 45147 Essen, Deutschland hans-ulrich.schildhaus@uk-essen.de

Interessenkonflikt. H.-U. Schildhaus gibt an, dass kein Interessenkonflikt besteht.

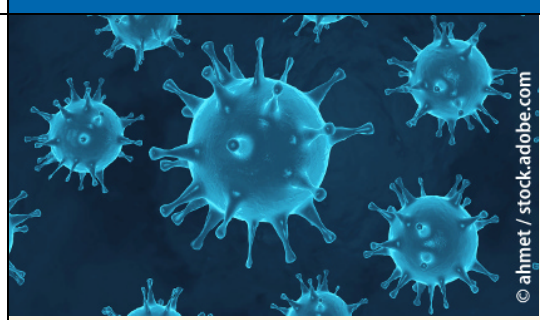

\section{Aktuelle Entwicklung zu COVID-19 bei Springer Nature und Springer Medizin}

Springer Nature und Springer Medizin unterstützen die globale Reaktion auf die COVID-19-Pandemie, indem ein schneller und direkter $\mathrm{Zu}$ gang zu den neuesten verfügbaren Forschungsergebnissen und Daten ermöglicht wird.

Auf der Homepage SpringerMedizin.de finden Sie ein immer aktuelles Dossier mit Beiträgen, Forschungsarbeiten und Ergebnissen zu SARS-CoV-2 sowie relevanten Links.

Darin z.B. auch die kürzlich publizierte

Empfehlung von DIVI, DGIIN, DGAI und DGP zur Intensivtherapie von Patienten mit COVID-19.

Springer Nature arbeitet mit globalen Organisationen zusammen, und verlinkt über SpringerNature.com/de auf eine eigene Landingpage mit einer Vielzahl an Information sowie freiem Zugriff auf die COVID-19-Contentplattformen von Nature Research, BioMed Central (BMC) und Springer.

Das Dossier zu Coronavirus / Covid-19 von Springer Medizin finden Sie hier: www.springermedizin.de/covid-19

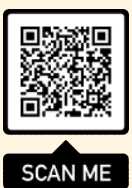

